\title{
Wireless Networks in Hospitals; a Framework of Use and Implementation for Nursing Care
}

\author{
Reza Safdari ${ }^{1}$ and Zahra Azadmanjir ${ }^{2}$ \\ ${ }^{1,2}$ Department of Health Information Management, Tehran University of Medical Sciences, Tehran, Iran \\ E-mail: 'rsafdari@tums.ac.ir, ${ }^{2} a z a d m @ r a z i . t u m s . a c . i r$
}

\begin{abstract}
The purpose of current study is presenting a framework for implementation of wireless networks in hospitals to use in nursing care. In the proposed framework, implementation key aspects that include the purpose, application domains, and the functions of wireless terminals, network technology, mobile devices, user interface and the information structure of the system have described in the logical framework. Because, the framework has presented based on scientific principles and the experiences of other countries, it can be an appropriate framework for future efforts in this field.
\end{abstract}

Keywords: Wireless Network, Hospital, Mobile Devices.

\section{INTRODUCTION}

Wireless technologies-based systems particularly mobile or handheld devices with clinical applications have a great potential to meet communication needs for medical and nursing staff in the today hospitals and the future electronic hospitals. These can be used by nurses in order to complete data communicating and processing duties in a faster and better way $[1,4]$.

In some of countries such as Finland, Germany, Canada, Brazil, South Korea and Taiwan, mobile devices used by nursing staff as wireless terminals or input-output part of large-scale information systems, for instance hospital information system, clinical information system (CIS) or nursing information system $[5,11]$.

Several studies conducted on the benefits of using from wireless or mobile terminals in nursing practice. These benefits are including following cases: (1) real-time electronic documentation and charting of patient care data (e.g. vital signs, assessments, observations, and body hydroelectric balance) in the bedside.[9-12] (2) timely retrieving of archived clinical data related to patients (e.g. health history, care plans, nursing notes, lab results) from central database.[9] [5] controlling of medicine administration based on physician orders for each of patients and completing of the medicine administration record in real-time that will be accountable for the decries of medication errors related to nursing performance.[12-16]

(4) decision support for nurses by mobile devices equipped to drug or medical reference applications and capabilities of alerting for drug interactions.[17] (5)clinical data exchanging in every time and setting.[4, 9, 18] (6) enhancing productivity of nursing staff $[2,19]$. Several projects can performed in around the world for develop and implement mobile-based applications that used by nurses but accomplishment of these in form of fully integrated in the hospitals is more complex than which are stand along. Nevertheless, there are different aspects in the process of development and implementation of wireless terminals in care practice which affecting on outcomes of that. For this reason, we studied different aspects through rational framework and examined the aspects in real examples available in some of countries for presenting of a framework for implementation.

\section{METHODS}

This research conducted in descriptivecomparative method. Information gathered from several sources. First, review of literature about wireless or mobile technology in nursing care by search in paper resources and electronic databases such as Pub Med, EBSCO and Ovid and so on. 
Second, comparative study has done in several countries that use from mobile terminals in nursing practice. The studied countries were Canada, Australia, England, Taiwan and South Korea. The purpose and application domain of the wireless nursing terminal system, type of selected network technology and mobile device for implementation and functions or capabilities compared. In addition, if possible, the structure of information in the system and the process of development studied. Selection of studied countries performed based on following reasons:

In America, Brazil and Canada have initiatives related to this high-tech solution. In Brazil handmobile device with integrated wireless network interface use to help nurses in the nursing process.[9] However, enough information is not available for present studies. In contrast, Canada has a nursing information system [5] that used in expended level in the country. The system equipped to wireless nursing charting technology [6]. Therefore, Canada selected.

In Oceania, Australia was selected because this technology developed by university projects in some of hospitals.[20-22]In Europe, England, Finland and Germany are active; however, only required information for this study has been exist on England. In this reason, this country has studied. In Asia, Taiwan and South Korea developed nursing wireless terminals in the some of their hospitals and information related to these has been available. Then both two countries have selected.

\section{RESULT}

The results of first section of present study showed that there are several issues on use of wireless technologies in healthcare and particularly nursing care, which should considered. They are include but not limited to following issues:

\subsection{Communication infrastructure}

Communication infrastructure described as telecommunication technologies, networks and protocols used. They are fundamental technical requirements which affect on capabilities of system.[23] Specifications and data transfer capacity in wireless network technologies should be considering according to aims of implementation and expected functions. In the present of time, most wireless technologies in care industry are wireless local area networks (WLANs), Bluetooth and ZigBee. The features of these network described in Table 1.[23, 24] According to Ammenwerth, due to good range and data transfer rate in WLANs and their potential for connect with existing wired local networks, they are proper networks in care.[4]

ZigBee in healthcare domain usually is appropriate technology in order to establish the wireless body area networks for transferring biomedical signals such as electrocardiography (ECG) and electroencephalography (EEG), from body sensors to bedside monitoring equipment.[24] Bluetooth often applied as a communicating method between wearable sensors and mobile phones, Personal digital assistant or laptops in form of wireless personal area network (WPAN) for telecare and telemedicine.[25, 26] For this reasons, two technologies, which noted, cannot provide most proper capabilities for nursing terminals.

Table 1: Specifications of Wireless Network Technologies in Healthcare

\begin{tabular}{|l|c|c|c|}
\hline $\begin{array}{l}\text { Network } \\
\text { Specificatio } \\
\text { ns }\end{array}$ & $\begin{array}{c}\text { WLANs } \\
\left(\mathrm{Wi}^{\star} \mathrm{Fi}^{\star}\right) \\
(\mathrm{IEEE802.11} \\
\mathrm{b} / \mathrm{g})\end{array}$ & $\begin{array}{c}\text { Bluetoo } \\
\text { th } \\
(\text { IEEE8 } \\
02.15 .1)\end{array}$ & $\begin{array}{c}\text { ZigBee } \\
(\text { IEEE8 } \\
02.15 .4 \\
)\end{array}$ \\
\hline $\begin{array}{l}\text { Frequency } \\
\text { band }\end{array}$ & $2.4 \mathrm{GHz}$ & $2.4 \mathrm{GHz}$ & $2.4 \mathrm{GHz}$ \\
\hline Bandwidth & $20 \mathrm{MHz}$ & $1 \mathrm{MHz}$ & $5 \mathrm{MHz}$ \\
\hline $\begin{array}{l}\text { Data } \\
\text { transfer } \\
\text { rate }\end{array}$ & $54 \mathrm{MBPs}$ & $\begin{array}{c}732- \\
733 \mathrm{~KB} \\
\text { Ps }\end{array}$ & $\begin{array}{c}250 \mathrm{~KB} \\
\text { Ps }\end{array}$ \\
\hline Range & $\begin{array}{c}50-100 \mathrm{~m} \\
\text { indoor } \\
100-500 \mathrm{~m} \\
\text { outdoor }\end{array}$ & $0-100 \mathrm{~m}$ & $0-30 \mathrm{~m}$ \\
& & \\
\hline
\end{tabular}

Wireless Fidelity

According to some researcher, nurses are often moving between bedside and nursing workstation in wards of the hospital for conducting of their duties such as care documentation and orders communication. For this reason, they stated that it seems that WLAN is proper network for connect between mobile devices used by nurses with central clinical database and other information systems in the hospital.[4, 27, 28]

\subsection{Device Type}

Personal digital assistant (PDA), tablet and laptop are three types of common handheld devices which used by care providers. Using from PDA in healthcare begin from 1990 and then gradually was popular device for timely access to information resources in clinical and educational domains of nursing.[29-32] Findings of several research showed that PDA is proper from functional aspect for documentation.[33] In contrast, other same studies showed, physical specifications, limited 
memory and small screen size, difficulty of data entry and low speed processor of PDA are problems for using from it by medical and nursing staff.[23, 34, 35] However, laptop has not limitations of PDA, but according to some of experts, tablet is the best alternative for PDA in care practices for documenting, retrieve and display of medical images in beside $[23,36]$.

\subsection{Interface configuration and data display}

It is refer to design of user interface and display various types of data such as text, image and multimedia data. In this regard, ergonomic design of used device, data entry methods, resolution, structure of screen and navigational capabilities are notable issues that will affect on adaptation of the system by nurses as end-users of nursing wireless terminals $[12,13,23,28,36]$.

\subsection{Purpose and functions of the system}

Wireless systems applied in different area of healthcare such as emergency settings for triage[21, 37, 38] or monitoring of patients physiological signals.[39] Purposes of using are various in depended on application domains. nursing application domain is extended from physiological condition of patients, documenting or charting to decision support and medication administration. [24, 40] Based on previous studies, documentation and medication therapy management are two aspects of nursing practice which will be utilized from wireless terminals more than other.[41]

\subsection{Security mechanism}

Wireless terminals as well as other information systems are in face to different kinds of security threats. In this reason, data protection and security of mobile or wireless technologies are key aspects in order to increase user acceptance of these in health industry. There are various standards and protocols or mechanisms to provide secure wireless networks. In this condition, will not easy to decision about selection of the best solutions. It is a hot topic that considered by research today. Marti and his colleagues in their study recommended proper security mechanisms based on the different levels of the wireless networks as following items:

Security in the data link layer provides hop-to hop protection by Bluetooth, Zigbee. Security in the network layer provides node-to node protection by IP security (IP sec). In the transport layer provides an end-to end protection. Secure Sockets Layer/ Transport Layer Security (SSL/TLS) or Hypertext Transfer Protocol over Secure Socket (HTTPS) are examples of security protocols in this layer. Also application layer security is provided through the encryption or signature of the data sent through the communications stack in form of application to application and application-user to application-user protection.[42, 43] Moreover, for provide a secure mobile devices can be used from biometric technology such as fingerprint capability or iris scan mechanism [44].

\section{NURSING WIRELESS TERMINALS IN HOSPITALS OF SELECTED COUNTRIES}

The comparative study indicated that in Canada, wireless nursing charting system developed as terminal of nursing information system [5] which used by nurses in order to real-time charting in bedside. Laptop is selected device used nurses for connected with central server of NIS through WLAN in hospital in order to loading archived patient data or recording and saving new data to the database [6].

In Australia, the wireless terminals have been implemented through various academic projects in order to facilitate nursing activities in some hospitals.[21, 22] At the Austin and Heidelberg hospitals, tablet used to wireless communicate with hospital information system [45] for unrestricted access to clinical data in wired local area networkbased hospital systems. This connection is established through a IEEE $802.11 \mathrm{~g}$ standard of wireless local area network [46].

Also in England, bedside wireless terminals in form of multi-purpose implemented in some of the NHS hospitals. These purposes are entertainment for patients and data entry by nurses as well as physicians to electronic patient records (EPR) through $802.11 \mathrm{~b} / \mathrm{g}$ wireless LAN in the bedside [47].

Mobile Nurse System in Korea as terminals designed to communicate with hospital information system [45] via mobile support system (MSS) which interchanges and stores clinical data. The type of network and mobile device were WLAN and PDA [7].

In Taiwan, mobile nursing information system designed to perform paperless recording of nursing information at the bedside. PDA-based terminals on wireless local area network connected with clinical database central server.[8]

The results of this study on performances and capabilities of nursing wireless terminals in selected countries showed in Table 2.

The results of study on implementation process of this technology showed that the system in Canada developed in modular design and Melfort 
hospital became the pilot site.[6] No more information exists in this area. In Australia, the system has designed following the survey on attitude of the nurses working in ICU and the oncology units. The physical characteristics and environmental conditions evaluated in order to define the infrastructures and selection of the network components. After prototype implementation, nursing staff trained in order to working with the terminals. Satisfaction of nurses on terminals performance and work flow in these were evaluated in six months after the implementation [22].

Table2 : Features of Wireless Terminals in The Countries

\begin{tabular}{|c|c|c|c|c|c|}
\hline \multirow[t]{2}{*}{ Item } & \multicolumn{5}{|c|}{ Studied countries } \\
\hline & ڤ్లి & 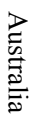 & 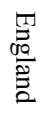 & 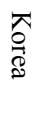 & . \\
\hline $\begin{array}{l}\text { A- Integrated to admission, } \\
\text { discharge, transfer system for } \\
\text { providing of patients list in } \\
\text { wards }\end{array}$ & $\sqrt{ }$ & $\sqrt{ }$ & $\sqrt{ }$ & $\sqrt{ }$ & $\sqrt{ }$ \\
\hline $\begin{array}{l}\text { B- Possibility of retrieving patients } \\
\text { clinical data }\end{array}$ & $\sqrt{ }$ & $\sqrt{ }$ & $\sqrt{ }$ & $\sqrt{ }$ & $\sqrt{ }$ \\
\hline $\begin{array}{l}\text { C- Capability of recording vital } \\
\text { signs \& input/output fluids data } \\
\text { in flow sheets }\end{array}$ & $\sqrt{ }$ & $\sqrt{ }$ & $\sqrt{ }$ & $\sqrt{ }$ & $\sqrt{ }$ \\
\hline $\begin{array}{l}\text { D- Access to care plan related to } \\
\text { patients \& completing its } \\
\text { components such as problems, } \\
\text { aims \& nursing interventions }\end{array}$ & $\sqrt{ }$ & $\sqrt{ }$ & $\sqrt{ }$ & $\sqrt{ }$ & $\sqrt{ }$ \\
\hline $\begin{array}{l}\text { E- Documenting nurses reports in } \\
\text { the nursing notes }\end{array}$ & $\sqrt{ }$ & $\sqrt{ }$ & $\sqrt{ }$ & $\sqrt{ }$ & $\sqrt{ }$ \\
\hline $\begin{array}{l}\text { F- Possibility of retrieving the } \\
\text { physician orders list related to } \\
\text { patients }\end{array}$ & - & $\sqrt{ }$ & $\sqrt{ }$ & $\sqrt{ }$ & - \\
\hline $\begin{array}{l}\text { G- Possibility of retrieving the } \\
\text { electronic medication record } \\
\text { and documenting drug used by } \\
\text { patient according to orders }\end{array}$ & - & - & $\sqrt{ }$ & $\sqrt{ }$ & - \\
\hline $\begin{array}{l}\text { H- Connecting to laboratory \& } \\
\text { radiology systems in the } \\
\text { hospital }\end{array}$ & $\sqrt{ }$ & $\sqrt{ }$ & $\sqrt{ }$ & $\sqrt{ }$ & $\sqrt{ }$ \\
\hline I- Display of discharge summery & _- & $\sqrt{ }$ & - & - & - \\
\hline $\begin{array}{l}\text { J- Equipped to drug or medical } \\
\text { reference applications }\end{array}$ & - & - & $\sqrt{ }$ & - & - \\
\hline K- Access to the Internet & _ & $\sqrt{ }$ & $\sqrt{ }$ & _- & - \\
\hline $\begin{array}{l}\text { L- Equipped to RFID }{ }^{\star} \text { technology } \\
\text { for control of drug } \\
\text { administration }\end{array}$ & - & - & $\sqrt{ }$ & - & - \\
\hline
\end{tabular}

Radio Frequency Identification

No information was available on the development process of the wireless bedside terminals in England. Development process of terminals in Korea was composed of following steps: daily nursing workflow analysis, define functions which can be facilitated by terminals, software and user interface design based on nurse's requirements analysis, prototype development and implementing of clinical trial in hospital of Seoul National University [7].The structure of software on PDA designed in form of multi-layers. It is include initial browser in first layer, general information, orders, nursing note, and nursing unit process in second layer and detailed records in subsequent layers [7]. The process in Taiwan was included workflow analysis to clearly understand nurses' daily work and to determine which nursing tasks could be appropriately perform on a PDA, to define user requirements, develop interface and prototype based on considerations of mobile human-computer interaction. Designed system implemented in a pilot site at hospital and its usability evaluated [8]. Moreover, the structure of system was included the three layers of identification, main functions and sub-functions [8].

\section{DISCUSSION}

Based on the research findings, all five countries use wireless local area network for establishment of connection between wired local network in the hospital and mobile or wireless terminals used by nurses. It is according to previous studies on proper wireless network technology for nursing practice $[4,27,28]$.

The findings of the study indicate that PDA is most popular mobile device, which used for bedside nursing charting which is similar to other researches on this issue [29-33].

Findings of the present research showed that connection with admission, discharge system of hospital, retrieving patient's clinical data, recording vital sign and input/output fluids data, access to care plan related to patients \& completing it, documenting nurse's reports in the nursing notes and connecting to laboratory and radiology systems are main and common functions of the terminals. In a research by Chang and et al, results showed all of respondent nurses $(\mathrm{n}=26)$ stated that quick access to patient information through mobile devices was very helpful.[48] results of the study of Lazarus showed in response to this statement "did the PDA device improve access to patient information at the bedside?" 23 cases have strongly agreed and 585 agreed.[49] Findings of present study will support from the research of Chang and Lazarus.

In addition, in the all of the countries where information on implementation process of their nursing wireless terminals was available; workflow analysis was one of first phases. In addition, evaluation of user's requirements in primary step and their satisfaction or views after system design has done in Korea and Taiwan. 
R. Safdari and Z. Azadmanjir / International Journal of Computer Networks and Communications Security, 2 (6), June 2014

Results on information structure of the system indicated that, general information relative to patient, orders list, nursing notes and lab results are minimum information components in mobile devices in selected countries.

Therefore, based on results of current study on most important aspects of successful implement of wireless technologies in nursing practice and experiences of several developed countries in this issue, the following framework proposed:

As a regard of key aspects of wireless systems implementation and best practices in selected countries, we are presenting a framework for implementing nursing wireless terminals in hospitals. Proposed framework is including following steps:

\subsection{Conceptual design of the system functions}

\subsubsection{Define of purposes and application domains:}

Because, expected purposes specify application domains of the terminals, in first step, they should be identifying. Based on former studies, improve the productivity of nursing staff in the hospital, $[2$, 19] facilitate the nursing time consuming of activities such as documentation and orders communication $[9,50]$ are some of the purposes of use wireless or mobile technologies. In addition, the enhancing of patient safety and care quality by decries of medication errors relevant to nurses performance, are other the purposes of use.[14, 51, 52]

With regard to these purposes, which can be considering for Iran, application domains are nursing documentation and medication administration.

\subsubsection{Analysis of workflow related to defined application domains for identify expected functions:}

In general, workflow for nursing documentation closely related to nursing process that include assessment, identify patient problems and diagnosis, identify expected outcomes, care plan, implementation and evaluation [53]. Moreover, nurse's duties about medication therapy management usually are in relation with drug administration and documentation. However, detailed workflow in each of facilities can be different. Therefore, it should exactly analyze.

\subsubsection{Define expected functions for terminals:}

Based on the results of comparative study, following functions have expected for nursing wireless terminals:

- Integrated to Admission, Discharge and Transfer system for access to patients list

- Connected with clinical database in the hospital

- Recording patient vital signs and input/output data in the bedside

- $\quad$ Access to care plan and completing it in the bedside

- Documenting nursing reports in nursing notes each patient

Retrieving physician orders relative to - $\quad$ Connected with laboratory and radiology information systems existing in the hospital

- Access to electronic medication record relative to each patients for documenting administrated drug data according to orders

- $\quad$ Access to drug references

\subsection{Conceptual design of the system structure}

\subsubsection{Determine data types that gathered or displayed by wireless terminal:}

As regard to defined functions, types of data will specified. Often, the type of data is text, unless connection to radiology systems in order to retrieving medical images or scanning medication or patient wristband barcodes defined as a function for the system.

\subsubsection{Selection of mobile device:}

In this stage, should be selected mobile device used as terminals based on defined functions and data types. In this issue, a framework has proposed in Table 3.

Table 3: The Proposed Framework for Selection of Mobile Device

\begin{tabular}{|l|c|}
\hline \multicolumn{1}{|c|}{ Function/data type } & Proposed device \\
\hline Documentation/ text only & $\begin{array}{c}\text { PDA-Tablet- } \\
\text { Laptop }\end{array}$ \\
\hline $\begin{array}{l}\text { Documentation/ text } \\
\text { Image display/ image }\end{array}$ & Tablet-Laptop \\
\hline $\begin{array}{l}\text { Documentation/ text } \\
\text { Scanning/ barcode }\end{array}$ & PDA $^{\star}$ \\
\hline
\end{tabular}

Personal Digital Assistant 


\subsubsection{Selection of communication network and description of network components architecture:}

According to previous studies by Ammenwerth [4] and other researches [27, 28] and also the results of comparative study recommended that "IEEE $802.11 \mathrm{~b} / \mathrm{g}$ or n" used for network development. However, survey should be conducted on environment in order to determine the required communication equipment configuration and proper location for install the Access Points, antenna and other components of the network.

\subsection{Technical design}

In present framework recommended to considering the ISO 13407 standard in technical design. It provides a framework for human-centered design processes for interactive systems that include specify the context of use, specify the user and organizational requirements, producing prototype and evaluate designs against user requirements[54].based on this standard, prior to technical design, users and other stockholders requirements should be analyzed. It may implement by interview or survey with nurses and others about the system expected functions and specifications of user interface and information structures in the software.

\subsubsection{Technical design of user interface in the terminals:}

In addition to users requirements analysis, should be attention to ergonomic principles in during of user interface design. In this reason, ISO 9241 standard used that originally titled "Ergonomic requirements for work with visual display terminals (VDTs)" and so called by "Ergonomics of humansystem interaction" now. It provides the comprehensive guidelines on design of layout, menus, icons, commend dialogues, error alerts, navigational structures and so on that can be helpful for increase usability of nursing wireless terminals and convenience of working with them.

\subsubsection{Technical design of information structure in terminals:}

Because nursing documentation is one main function of the wireless terminals, modules and electronic forms available at them should be organized in way that data retrieving and navigation in different sections easy. Best approach is constitutes sections in different layers as well as Korea and Taiwan.
Therefore, recommended that information components in the software of terminals should be organized as follows:

First layer: it is home or entrance page that have three parts includes authentication and log in, the system help and medication reference.

- $\quad$ Second layer: it wills accessible after log in and include patient list hospitalized in clinical departments of the hospital. By selection of each patient, user can be access to information relative to selected patient that is available in next layer.

Third layer: include general information related to patient, health history, nursing record and physician orders.

- $\quad$ Fourth layer: this layer is including sections of nursing record that completed by nurses such as vital sign and input/output flow sheets, nursing care plan, nursing notes and medication administration record.

\subsection{Implementation}

According to ISO 13407, after technical design, the prototype system should be offered to users in small scale (pilot site can be one department of the hospital). In this stage, the emphasis will be on obtaining feedback to detect of possible defects, which can be used to improve the design before full implementation. Time response, usability, compatibility with other systems in the hospital and user-friendly of interface should be evaluated. It is a formative evaluation, which is essential to reduce the financial losses due to defects and possible rejection of the new technology by users. After installation and full implementation, nursing staff should receive train on how work with the system. In addition, summative evaluation is essential to assess whether the purposes have achieved.

In the present framework, tried to implementation key aspects that include the purpose, application domains, the functions of wireless terminals, network type, mobile devices, user interface and information structure of the system described in the logical framework. Emphasis on the technical design, based on ISO 13407 and ISO 9241 standards are the notable features of the proposed framework. Finally, because the proposed framework in this study presented based on scientific principles and the experiences of other countries, it seems, that is an appropriate framework for future efforts in this field.

\section{REFERENCES}

[1] K. Rempher, C. Lasome, and T. Lasome, "Leveraging palmtechnology in the advanced 
R. Safdari and Z. Azadmanjir / International Journal of Computer Networks and Communications Security, 2 (6), June 2014

practice nursing environment.," Adv Crit Care, vol. 14, pp. 363-370, 2003.

[2] A. Craig, "Personal digital assistant use: practical advice for the advanced practice nurse," Topics in Advanced Practice Nursing, vol. 2, pp. 6-8, 2002.

[3] R. L. Simpson, "Wireless communications, a new frontier," J Nurs Manag, vol. 27, pp. 2021, 1996.

[4] E. Ammenwerth, A. Buchauer, B. Bludau, and R. Haux, "Mobile information and communication tools in the hospital," Int J Med Inform, vol. 57, pp. 21-40, 2000.

[5] E. Kimura, Y. Onozaki, Y. Shizui, and M. Ohnishi3, "Development of Nursing Care Support System using PDA with wireless and barcode reader," AMIA 2003 Symposium Proceedings, p. 894, 2003.

[6] B. K. Scott, "Rural Hospital Pioneers Wireless Nurse Charting System," Official Journal of canadian health informatics association, vol. 27, pp. 16-18, April 2003.

[7] J. Choi, J. Chun, K. Lee, S. Lee, D. Shin, S. Hyun, et al., "MobileNurse: hand-held information system for point of nursing care," Comput Methods Programs Biomed, vol. 74, pp. 245-254, 2004.

[8] K.-W. Su and C.-L. Liu, "A Mobile nursing information system based on human-computer interaction design for improving quality of nursing," J Med Syst [Epub ahead of print], Sep 2010.

[9] D. Sperandio and Y. Evora, "The use of mobile computational technology in the nursing process: a new challenge for Brazilian nurses," Stud Health Technol Inform, vol. 146, p. 721, 2009.

[10] S. B. Rosales, M. Mueller-Gorchs, and C. Kunze, "Scenario-based design of an ICT platform for mobile information services in ambulatory care nursing," Stud Health Technol Inform, vol. 146, p. 64, 2009.

[11] S. Malmqvist, "Using electronic patient record with PDA, benefits and nursing staffs' knowhow to use technology," Stud Health Technol Inform, vol. 146, p. 709, 2009.

[12]L. Nelson, "Technology: nursing the system. Step-by-step guide to selecting mobile wireless devices," J Nurs Manag, vol. 30, pp. 12-3, Nov 1999.

[13]B. Garrett and G. Klein, "Value of wireless personal digital assistants for practice: perceptions of advanced practice nurses," J Clin Nurs, vol. 17, pp. 2146-2154, 2008.

[14]K. Galt, A. Rule, B. Houghton, D. Young, and G. Remington, "Personal digital assistant-based information sources: potential to improve medication safety. ," J Med Libr Assoc, vol. 93, pp. 229-236, 2005.

[15] M. Russo, "eMAR and mobile computing: why nursing homes need to get wired now," Nursing Homes: Long Term Care Management vol. 57, pp. 32-4, Jan 2008.

[16] P. E. Johansson, G. I. Petersson, and G. C. Nilsson, "Personal digital assistant with a barcode reader-A medical decision support system for nurses in home care," International Journal of Medical Informatics, vol. 79, pp. 232-242, 4// 2010.

[17]C. M. Ruland, "Handheld Technology to Improve Patient Care: Evaluating a Support System for Preferencebased Care Planning at the Bedside," Am Med Inform Assoc, vol. 9, pp. 192-201, Mar / Apr 2002.

[18] S. Breslin and W. Greskovich, "Wireless Technology Improves Nursing Workflow and Communications," Comput Inform Nurs, vol. 22 pp. 275-281, September-October 2004.

[19]M. Tooey and A. Mayo, "Handheld technologies in a clinical setting," AACN Adv Crit Care, vol. 14, pp. 342-349, 2003.

[20] Introduction to ISO 13407. Available: http://www.ucc.ie/hfrg/emmus/methods/iso.ht $\mathrm{ml}$

[21]R. Gururajan, "A study of the use of hand-held devices in an emergency department," J Telemed Telecare, vol. 10 Suppl 1, pp. 33-5, 2004.

[22](2011, August 12). HP Nursing Project in Royal Women's Hospital. Available: http://www.cs.rmit.edu.au/MobileComputing/p da.html

[23]P. Olla and J. Tan, "The M-Health Reference Model: An Organizing Framework for Conceptualizing Mobile Health Systems," in Medical Informatics:Concepts, Methodologies, Tools, and Applications, J. Tan, Ed., ed Hershey, New York, 2009, pp. 455-467.

[24] M. R. Yuce, "Implementation of wireless body area networks for healthcare systems," Sensors and Actuators, vol. 162, pp. 116-129, 2010.

[25] H. Ding, D. Ireland, R. Jayasena, J. Curmi, and M. Karunanithi, "Integrating a mobile health setup in a chronic disease management network," Stud Health Technol Inform, vol. 188, pp. 20-25, 2013.

[26] C. W. Jang, Y. T. Byun, T. Lee, D. H. Woo, S. Lee, and Y. M. Jhon, "A Wireless Monitoring Sub-nA Resolution Test Platform for Nanostructure Sensors," Sensors (Basel), vol. 13, pp. 7827-37, 2013. 
R. Safdari and Z. Azadmanjir / International Journal of Computer Networks and Communications Security, 2 (6), June 2014

[27]K. Banitsas, R. S. H. Istepanian, and S.Tachakra, "Applications of medical wireless LAN systems," Int. J. Med. Mark, vol. 2, pp. 136-143, 2002.

[28] S.-J. Hsiao, Y.-C. Li, Y.-L. Chen, and H.-C. Ko, "Critical Factors for the Adoption of Mobile Nursing Information Systems in Taiwan: the Nursing Department Administrators' Perspective," J Med Syst, vol. 33, pp. 369-377, 2009.

[29] M. Farrell, I. Mcgrath, M. D'arcy, and E. Abaloz, "Improving patient health outcomes in acute care hospital units using mobile wireless technology and handheld computers," Comput Inform Nurs, vol. 25, pp. 308-309, 2007.

[30]M. J. Farrell and L. Rose, "Use of Mobile Handheld Computers in Clinical Nursing Education," J Nurs Educ, vol. 47, pp. 13-19, January 2008.

[31]K. Rosenthal, "'Touch' vs. 'tech': valuing nursing-specific PDA software " J Nurs Manag, vol. 34, pp. 58-60, 2003.

[32] J. Miller, J. Shaw-Kokot, M. Arnold, T. Boggin, K. Crowell, F. Allegri, et al., "A study of personal digital assistants to enhance undergraduate clinical nursing education," J Nurs Educ, vol. 44, pp. 19-26, 2005.

[33] M. L. Zalon, S. Sandhaus, D. Valenti, and U. Arzamasova, "Using PDAs to detect cognitive change in the hospitalized elderly patient," Appl Nurs Res, vol. 23, pp. e21-e27, 2010.

[34] S. Fischer, T. E. Stewart, S. Mehta, R.Wax, and S. E. Lapinsky, "Handheld computing in medicine," J Am Med Inform Assoc, vol. 10, pp. 139-149, 2003.

[35] M. F. T. Peterson, "The right information at the point of care library delivery via hand-held computers," presented at the the EAHIL Conference of Health and Medical Libraries, Cologne, 2002.

[36] L. Heslop, S. Weeding, L. Dawson, J. Fisher, and A. Howard, "Implementation Issues for Mobile-Wireless Infrastructure and Mobile Health Care Computing Devices for a Hospital Ward Setting," J Med Syst, vol. 34, pp. 509518, 2010.

[37] W. Michalowski, R. Slowinski, S. Wilk, K. J. Farion, J. Pike, and S. Rubin, "Design and Development of a Mobile System for Supporting Emergency Triage," Methods Inf Med, vol. 44, pp. 14-24, 2010.

[38] O. Väisänen, M. Mäkijärvi, and T. Silfvast, "Prehospital ECG transmission: comparison of advanced mobile phone and facsimile devices in an urban Emergency Medical Service
System " Resuscitation, vol. 57, pp. 179-185, May 2003.

[39]E. Jovanov, D. Raskovic, J. Price, A. Krishnamurthy, J. Chapman, and A. Moore, "Patient monitoring using personal area networks of wireless intelligent sensors," Biomed Sci Instrum, vol. 37, pp. 373-378, 2001.

[40]A. E. Carroll, S. Saluja, and P. TarczyHornoch, "The implementation of a Personal Digital Assistant (PDA) based patient record and charting system: lessons learned," Proc AMIA Symp, pp. 111-5, 2002.

[41] N. C. Nelson, R. S. Evans, M. Samore, and R. M. Gardner, "Detection and Prevention of Medication Errors Using Real-Time Bedside Nurse Charting," Journal of the American Medical Informatics Association vol. 12, Jul / Aug 2005.

[42]R. Martí and J. Delgado. (2003, Jun 21). Security in a Wireless Mobile Health Care System. Available: http://www2.research.att.com/ rjana/MartiDelgado.pdf

[43]R. Martí, J. Delgado, and X. Perramon, "Security specification and implementation for mobile e-health services," in International Conference on e-Technology, e-Commerce and e-Service :EEE '04, 2004, pp. 241-248.

[44]P. Pharow and B. Blobel, "Mobile health requires mobile security: Challenges, solutions, and standardization," Stud Health Technol Inform, vol. 136, p. 697, 2008.

[45] R. S. H. Istepanian, S. Laxminarayan, and C. Pattichis, Eds., M-health: Emerging mobile health systems. London: Springer-Verlag, 2006, p.^pp. Pages.

[46] V. Moritz, "Wireless technology implementation,Pilot projects in ICU and Ward 5: Report for HealthSMART by Austin Health," Austin Health, Victoria, Australia 2011.

[47]HTS. (2011, September 15). HTS Patient InfotainmentTerminals Hospicom 3. Available: http://www.hospicom.co.uk

[48] K. Chang, K. D. Lutes, M. L. Braswell, and J. K. Nielsen, "Nurses' Perceptions of Using a Pocket PC for Shift Reports and Patient Care," in Medical informatics : concepts, methodologies, tools, and applications, J. Tan, Ed., ed Hershey. USA: Information Science Reference, 2009, pp. 799-810.

[49] S. S. Lazarus, "The Evaluation of Wireless Devices Used by Staff at Westmead Hospital," in Medical informatics : concepts, methodologies, tools, and applications, J. Tan, 
Ed., ed Hershey.USA: Information Science Reference, 2009, pp. 811-821.

[50]B. Garrett and C. Jackson, "Mobile clinical learning using wireless personal digital assistants for professional healthcare students," The International Journal of Learning, vol. 13, pp. 135-144, 2006.

[51]E. Chen, E. Mendonca, L. McKnight, P. Stetson, J. Lei, and J. Cimino, "PalmCIS: a wireless handheld application for satisfying clinician information needs," J Am Med Inform Assoc, vol. 11, pp. 19-28, 2004.

[52]C. Honeybourne, S. Sutton, and L. Ward, "Knowledge in the palm of your hands: PDAs in the clinical setting," Health Info Libr J, vol. 23, pp. 51-59, 2006.

[53] K. K. Nahid and T. hasantehrani, "A Survey Comparison Quality of Writing and Application Nursing Process Between Students of Hamadan City, 2010," Procedia - Social and Behavioral Sciences, vol. 31, pp. 762-767, 2012.

[54] J. Earthy, B. S. Jones, and N. Bevan, "The improvement of human-centred processes-facing the challenge and reaping the benefit of ISO 13407," Int J Hum Comput Stud, vol. 55, pp. 553-585, 2001. 